\title{
NON-ENERGY SEMI-STABLE RADIAL SOLUTIONS
}

\author{
SALVADOR VILLEGAS
}

\begin{abstract}
This paper is devoted to the study of semi-stable radial solutions $u \notin H^{1}\left(B_{1}\right)$ of $-\Delta u=f(u)$ in $\overline{B_{1}} \backslash\{0\}=\left\{x \in \mathbb{R}^{N}: 0<\right.$ $|x| \leq 1\}$, where $f \in C^{1}(\mathbb{R})$ and $N \geq 2$. We establish sharp pointwise estimates for such solutions. In addition, we prove that in dimension $N=2$, any semi-stable radial weak solution of $-\Delta u=f(u)$, posed in $B_{1}$ with Dirichlet data $\left.u\right|_{\partial B_{1}}=0$, is regular.
\end{abstract}

\section{InTRODUCTION AND MAIN RESULTS}

This paper deals with the semi-stability of radial solutions of

$$
-\Delta u=f(u) \text { in } \overline{B_{1}} \backslash\{0\},
$$

where $B_{1}$ is the open unit ball of $\mathbb{R}^{N}, N \geq 2$ and $f \in C^{1}(\mathbb{R})$. We consider classical solutions $u \in C^{2}\left(\overline{B_{1}} \backslash\{0\}\right)$. This is not a restriction. In fact, if we consider a radial solution $u$ of this equation in a very weak sense, we obtain that $u$ is a $C^{3}$ function.

A solution $u$ of (1.1) is called semi-stable if

$$
Q_{u}(v):=\int_{B_{1}}\left(|\nabla v|^{2}-f^{\prime}(u) v^{2}\right) d x \geq 0
$$

for every $v \in C^{1}\left(B_{1}\right)$ with compact support in $B_{1} \backslash\{0\}$. Formally, the above expression is the second variation of the energy functional associated to (1.1) in a domain $\Omega \subset \subset B_{1} \backslash\{0\}: E_{\Omega}(u)=\int_{\Omega}\left(|\nabla u|^{2} / 2-F(u)\right) d x$, where $F^{\prime}=f$. Thus, if $u \in C^{1}\left(\overline{B_{1}} \backslash\{0\}\right)$ is a local minimizer of $E_{\Omega}$ for every smooth domain $\Omega \subset \subset B_{1} \backslash\{0\}$ (i.e. a minimizer under every small enough $C^{1}(\bar{\Omega})$ perturbation vanishing on $\partial \Omega$ ), then $u$ is a semi-stable solution of (1.1).

We will be also interested in the semi-stability of radial weak solutions of the problem

$$
\begin{cases}-\Delta u=f(u) & \text { in } B_{1} \\ u=0 & \text { on } \partial B_{1}\end{cases}
$$

where $N \geq 2$ and $f \in C(\mathbb{R})$.

As in [1], we say that $u$ is a weak solution of (1.2) if $u \in L^{1}\left(B_{1}\right), f(u) \delta \in$ $L^{1}\left(B_{1}\right)$ and

The author has been supported by the MEC Spanish grant MTM2012-37960. 


$$
-\int_{B_{1}} u \Delta \zeta d x=\int_{B_{1}} f(u) \zeta d x
$$

for all $\zeta \in C^{2}\left(\overline{B_{1}}\right)$ with $\zeta=0$ on $\partial B_{1}$. Here $\delta(x)=\operatorname{dist}\left(x, \partial B_{1}\right)$ denotes the distance to the boundary of $B_{1}$.

If $f \in C^{1}(\mathbb{R})$, we say that a radial weak solution $u$ of $(1.2)$ is semi-stable if $\left.u\right|_{\overline{B_{1}} \backslash\{0\}}$ is semi-stable. This definition has sense, since any radial weak solution of (1.2) is a $C^{2}\left(\overline{B_{1}} \backslash\{0\}\right)$ function (see Lemma 3.1 below).

The original motivation of this work is the following. Consider the semilinear elliptic problem

$$
\begin{cases}-\Delta u=\lambda g(u) & \text { in } \Omega, \\ u \geq 0 & \text { in } \Omega, \\ u=0 & \text { on } \partial \Omega,\end{cases}
$$

where $\Omega \subset \mathbb{R}^{N}$ is a smooth bounded domain, $N \geq 1, \lambda \geq 0$ is a real parameter and the nonlinearity $g:[0, \infty) \rightarrow \mathbb{R}$ satisfies

$$
g \text { is } C^{1} \text {, nondecreasing and convex, } g(0)>0 \text {, and } \lim _{u \rightarrow+\infty} \frac{g(u)}{u}=+\infty \text {. }
$$

It is well known that there exists a finite positive extremal parameter $\lambda^{*}$ such that $\left(P_{\lambda}\right)$ has a minimal classical solution $u_{\lambda} \in C^{2}(\bar{\Omega})$ if $0 \leq \lambda<\lambda^{*}$, while no solution exists, even in the weak sense (similar definition as the case $\Omega=B_{1}$ ), for $\lambda>\lambda^{*}$. The set $\left\{u_{\lambda}: 0 \leq \lambda<\lambda^{*}\right\}$ forms a branch of classical solutions increasing in $\lambda$. Its increasing pointwise limit $u^{*}(x):=$ $\lim _{\lambda \uparrow \lambda^{*}} u_{\lambda}(x)$ is a weak solution of $\left(P_{\lambda}\right)$ for $\lambda=\lambda^{*}$, which is called the extremal solution of $\left(P_{\lambda}\right)$ (see [1, 2, 8]).

The regularity and properties of extremal solutions depend strongly on the dimension $N$, domain $\Omega$ and nonlinearity $g$. When $g(u)=e^{u}$, it is known that $u^{*} \in L^{\infty}(\Omega)$ if $N<10$ (for every $\Omega$ ) (see [7, 11]), while $u^{*}(x)=$ $-2 \log |x|$ and $\lambda^{*}=2(N-2)$ if $N \geq 10$ and $\Omega=B_{1}$ (see [9]). There is an analogous result for $g(u)=(1+u)^{p}$ with $p>1$ (see 2]). Brezis and Vázquez [2] raised the question of determining the boundedness of $u^{*}$, depending on the dimension $N$, for general nonlinearities $g$ satisfying (1.4). The first general results were due to Nedev [12, who proved that $u^{*} \in L^{\infty}(\Omega)$ if $N \leq 3$, and $u^{*} \in L^{p}(\Omega)$ for every $p<N /(N-4)$, if $N \geq 4$. In a recent paper the author [14] has proved that $u^{*} \in L^{\infty}(\Omega)$ if $N=4$, and $u^{*} \in L^{N /(N-4)}(\Omega)$, if $N \geq 5$. Cabré [3], proved that $u^{*} \in L^{\infty}(\Omega)$ if $N \leq 4$ and $\Omega$ is convex (no convexity on $f$ is imposed). If $N \geq 5$ and $\Omega$ is convex Cabré and Sanchón [6] have obtained that $u^{*} \in L^{\frac{2 N}{N-4}}(\Omega)$ (again, no convexity on $f$ is imposed). On the other hand, Cabré and Capella 4 have proved that $u^{*} \in L^{\infty}(\Omega)$ if $N \leq 9$ and $\Omega=B_{1}$. Recently, Cabré and Ros-Oton [5] have obtained that 
$u^{*} \in L^{\infty}(\Omega)$ if $N \leq 7$ and $\Omega$ is a convex domain of double revolution (see 5] for the definition).

Another interesting question is whether the extremal solution lies in the energy class. Nedev [12, 13] proved that $u^{*} \in H_{0}^{1}(\Omega)$ if $N \leq 5$ (for every $\Omega$ ) or $\Omega$ is convex (for every $N \geq 1$ ). The author [14 has obtained that $u^{*} \in H_{0}^{1}(\Omega)$ if $N=6$ (for every $\Omega$ ). Brezis and Vázquez [2] proved that a sufficient condition to have $u^{*} \in H_{0}^{1}(\Omega)$ is that $\liminf _{u \rightarrow \infty} u g^{\prime}(u) / g(u)>1$ (for every $\Omega$ and $N \geq 1$ ).

Note that the minimality of $u_{\lambda}\left(0<\lambda<\lambda^{*}\right)$ implies its semi-stability, i.e. $\int_{\Omega}\left(|\nabla v|^{2}-\lambda g^{\prime}\left(u_{\lambda}\right) v^{2}\right) d x \geq 0$, for every $v \in C^{1}(\Omega)$ with compact support. Clearly, we can pass to the limit and obtain that $u^{*}$ is also a semi-stable weak solution for $\lambda=\lambda^{*}$. Conversely, in [2] it is proved that if $g$ satisfies (1.4) and $u \in H_{0}^{1}(\Omega)$ is an unbounded semi-stable weak solution of $\left(P_{\lambda}\right)$ for some $\lambda>0$, then $u=u^{*}$ and $\lambda=\lambda^{*}$. (For instance, applying this result it follows easily that $u^{*}(x)=-2 \log |x|$ and $\lambda^{*}=2(N-2)$ if $g(u)=e^{u}$, $\Omega=B_{1}$ and $\left.N \geq 10\right)$. The hypothesis $u \in H_{0}^{1}(\Omega)$ is essential since in [2] it is observed that if $\Omega=B_{1}, N \geq 3$ and $\frac{N}{N-2}<p \leq \frac{N+2 \sqrt{N-1}}{N+2 \sqrt{N-1}-4}$, then $u(x)=|x|^{-2 /(p-1)}-1$ is an unbounded semi-stable weak solution of $\left(P_{\lambda}\right)$ for $g(u)=(1+u)^{p}$ and $\lambda=2(N p-2 p-N) /(p-1)^{2}$, which is a non-energy function, i.e. $u \notin H_{0}^{1}\left(B_{1}\right)$. Since $B_{1}$ is a convex domain, $u^{*} \in H_{0}^{1}\left(B_{1}\right)$ and then $u \not \equiv u^{*}$. As pointed out in [2], this type of "strange" solutions are apparently isolated objects that cannot be obtained as limit of classical solutions, which leaves them in a kind of "limbo" with respect to the classical theory.

In this paper we study this class of non-energy semi-stable radial solutions and it is established sharp pointwise estimates for such solutions. In addition we prove that, contrary to the case $N \geq 3$, there is no solutions of this type in dimension $N=2$.

Theorem 1.1. Let $N \geq 2, f \in C^{1}(\mathbb{R})$ and $u \notin H^{1}\left(B_{1}\right)$ be a semi-stable radial solution of (1.1). Then there exist $M>0$ and $0<r_{0}<1$ such that

$$
|u(r)| \geq \begin{cases}M|\log r| & \forall r \in\left(0, r_{0}\right) \text { if } N=2 \\ M r^{-N / 2-\sqrt{N-1}+2} & \forall r \in\left(0, r_{0}\right) \text { if } N \geq 3\end{cases}
$$

Theorem 1.2. Let $N \geq 2,0 \leq f \in C^{1}(\mathbb{R})$ and $u \notin H^{1}\left(B_{1}\right)$ be a semi-stable radially decreasing near the origin solution of (1.1). We have that:

i) If $N=2$, then $\lim _{r \rightarrow 0} r u^{\prime}(r)=-\alpha$, for some $\alpha \in(0,+\infty)$. In particular $\lim _{r \rightarrow 0} u(r) /|\log r|=\alpha$.

ii) If $N \geq 3$, then $M_{1} r^{-N / 2-\sqrt{N-1}+1} \leq\left|u_{r}(r)\right| \leq M_{2} r^{-N+1}$ in $\overline{B_{1}}$, for some constants $M_{1}, M_{2}>0$. 
Theorem 1.3. Let $N=2, f \in C^{1}(\mathbb{R})$ and $u$ be a semi-stable radial weak solution of (1.2). Then $u$ is regular (i.e. $u \in C^{2}\left(\overline{B_{1}}\right)$ ).

The main results obtained in this paper are optimal. If $N=2$, clearly $u(r)=|\log r| \notin H^{1}\left(B_{1}\right)$ satisfies $-\Delta u=0$ and then it is a semi-stable radial solution of (1.1) for $f \equiv 0$. On the other hand, for every $N \geq 2$ and $\alpha<0$ consider the radial function $u_{\alpha}(r)=r^{\alpha}, 0<r \leq 1$ and a function $f_{\alpha} \in C^{\infty}(\mathbb{R})$ satisfying $f_{\alpha}(s)=-\alpha(\alpha+N-2) s^{1-2 / \alpha}$ for every $s \geq 1$. If $N \geq 3$ and $2-N \leq \alpha<0$ then we take $f_{\alpha} \geq 0$. The following example shows that the pointwise estimates of Theorems 1.1 and 1.2 are sharp.

Example 1.4. Let $\alpha<0$ if $N=2$ and $\alpha \leq-N / 2-\sqrt{N-1}+2$ if $N \geq 3$. Consider the above defined functions $u_{\alpha}, f_{\alpha}$. Then $u_{\alpha} \notin H^{1}\left(B_{1}\right)$ is a semistable radial solution of (1.1) for $f=f_{\alpha}$.

Proof. It is immediate that $u_{\alpha} \notin H^{1}\left(B_{1}\right)$ is a radial solution of (1.1) for $f=$ $f_{\alpha}$. An easy computation shows that $f_{\alpha}^{\prime}\left(u_{\alpha}(r)\right)=-(\alpha-2)(\alpha+N-2) / r^{2}$, for every $0<r \leq 1$. Taking into account that $\alpha<0$ if $N=2$, and $\alpha \leq-N / 2-\sqrt{N-1}+2$ if $N=3$, we check at once that $-(\alpha-2)(\alpha+$ $N-2) \leq(N-2)^{2} / 4$, which is the best constant in Hardy's inequality: $\int_{B_{1}}\left((N-2)^{2} /\left(4 r^{2}\right)\right) v^{2} \leq \int_{B_{1}}|\nabla v|^{2}$, for every $v \in C^{1}\left(B_{1}\right)$ with compact support in $B_{1} \backslash\{0\}$. This gives the semi-stability of $u_{\alpha}$ for this range of values of $\alpha$.

\section{Sharp POINTWiSe ESTimates}

Lemmas 2.1 and 2.2 below are almost identical to Lemmas 2.1 and 2.2 of [15. We prefer to state them here and give the same proof as in [15] for the convenience of the reader. In fact, Lemma 2.1 follows easily from the ideas of the proof of [4, Lem. 2.1], which was inspired by the proof of Simons theorem on the nonexistence of singular minimal cones in $\mathbb{R}^{N}$ for $N \leq 7$ (see [10, Th. 10.10] and [4, Rem. 2.2] for more details).

Lemma 2.1. Let $N \geq 2, f \in C^{1}(\mathbb{R})$ and $u$ be a semi-stable radial solution of (1.1). Let $0<r_{1}<r_{2}<1$ and $\eta \in C^{0,1}\left(\left[r_{1}, r_{2}\right]\right)$ such that $\eta u_{r}$ vanishes at $r=r_{1}$ and $r=r_{2}$. Then

$$
\int_{r_{1}}^{r_{2}} r^{N-1} u_{r}^{2}\left(\eta^{2}-\frac{N-1}{r^{2}} \eta^{2}\right) d r \geq 0 .
$$

Proof. First of all, note that we can extend the second variation of energy $Q_{u}$ to the set of functions $v \in C^{0,1}\left(B_{1}\right)$ with compact support in $B_{1} \backslash\{0\}$, obtaining $Q_{u}(v) \geq 0$ for such functions $v$. Hence, we can take the radial function $v=\eta u_{r} \chi_{B_{r_{2}} \backslash \overline{B_{r_{1}}}}$.

On the other hand, differentiating (1.1) with respect to $\mathrm{r}$, we have

$$
-\Delta u_{r}+\frac{N-1}{r^{2}} u_{r}=f^{\prime}(u) u_{r}, \quad \text { for all } r \in(0,1) .
$$


Following the ideas of the proof of [4, Lem. 2.1], we can multiply this equality by $\eta^{2} u_{r}$ and integrate by parts in the annulus of radii $r_{1}$ and $r_{2}$ to obtain

$$
\begin{gathered}
0=\int_{B_{r_{2}} \backslash \overline{B_{r_{1}}}}\left(\nabla u_{r} \nabla\left(\eta^{2} u_{r}\right)+\frac{N-1}{r^{2}} u_{r} \eta^{2} u_{r}-f^{\prime}(u) u_{r} \eta^{2} u_{r}\right) d x \\
=\int_{B_{r_{2}} \backslash \overline{B_{r_{1}}}}\left(\left|\nabla\left(\eta u_{r}\right)\right|^{2}-f^{\prime}(u)\left(\eta u_{r}\right)^{2}\right) d x-\int_{B_{r_{2}} \backslash \overline{B_{r_{1}}}} u_{r}^{2}\left(|\nabla \eta|^{2}-\frac{N-1}{r^{2}} \eta^{2}\right) d x \\
=Q_{u}\left(\eta u_{r} \chi_{B_{r_{2}} \backslash \overline{B_{r_{1}}}}\right)-\omega_{N} \int_{r_{1}}^{r_{2}} r^{N-1} u_{r}^{2}\left(\eta^{\prime 2}-\frac{N-1}{r^{2}} \eta^{2}\right) d r .
\end{gathered}
$$

Using the semi-stability of $u$ the lemma follows.

Lemma 2.2. Let $N \geq 2, f \in C^{1}(\mathbb{R})$ and $u$ be a nonconstant semi-stable radial solution of (1.1). Then $u_{r}$ vanishes at most in one value in $(0,1)$.

Proof. Suppose by contradiction that there exist $0<r_{1}<r_{2}<1$ such that $u_{r}\left(r_{1}\right)=u_{r}\left(r_{2}\right)=0$. Taking $\eta \equiv 1$ in the previous lemma, we obtain

$$
\int_{r_{1}}^{r_{2}} r^{N-1} u_{r}^{2}\left(-\frac{N-1}{r^{2}}\right) d r \geq 0
$$

Hence we conclude that $u_{r} \equiv 0$ in $\left[r_{1}, r_{2}\right]$, which clearly forces $u$ is constant in $\overline{B_{1}} \backslash\{0\}$, a contradiction.

Lemma 2.3. Let $N \geq 2, u \in C^{2}\left(\overline{B_{1}} \backslash\{0\}\right)$ a radial function satisfying $u \notin H^{1}\left(B_{1}\right)$. Then there exist $0<a<1 / 2$ and a function $\eta_{0} \in C^{0,1}([a, 1 / 2])$ such that $\eta_{0}(a)=1, \eta_{0}(1 / 2)=0$ and

$$
\int_{a}^{1 / 2} r^{N-1} u_{r}^{2}\left(\eta_{0}^{\prime 2}-\frac{N-1}{r^{2}} \eta_{0}^{2}\right) d r<0
$$

Proof. For arbitrary $a \in(0,1 / 4)$ define the function

$$
\eta_{0}(r)= \begin{cases}1 & \text { if } a \leq r<1 / 4, \\ 2-4 r & \text { if } 1 / 4 \leq r \leq 1 / 2 .\end{cases}
$$

Clearly $\eta_{0}$ is a $C^{0,1}([a, 1 / 2])$ function satisfying $\eta_{0}(a)=1$ and $\eta_{0}(1 / 2)=0$. On the other hand

$$
\begin{gathered}
\int_{a}^{1 / 2} r^{N-1} u_{r}^{2}\left(\eta_{0}^{\prime 2}-\frac{N-1}{r^{2}} \eta_{0}^{2}\right) d r=-(N-1) \int_{a}^{1 / 4} r^{N-3} u_{r}^{2} d r \\
+\int_{1 / 4}^{1 / 2} r^{N-1} u_{r}^{2}\left(16-\frac{N-1}{r^{2}}(2-4 r)^{2}\right) d r
\end{gathered}
$$


Note that $u \in C^{2}\left(\overline{B_{1}} \backslash\{0\}\right)$ and $u \notin H^{1}\left(B_{1}\right)$ imply $r^{N-1} u_{r}^{2} \notin L^{1}(0,1 / 4)$ and therefore $\int_{0}^{1 / 4} r^{N-3} u_{r}^{2} d r=+\infty$. From the above it follows that

$$
\lim _{a \rightarrow 0} \int_{a}^{1 / 2} r^{N-1} u_{r}^{2}\left(\eta_{0}^{\prime 2}-\frac{N-1}{r^{2}} \eta_{0}^{2}\right) d r=-\infty .
$$

Taking $a \in(0,1 / 4)$ sufficiently small the lemma follows.

Lemma 2.4. Let $N \geq 2, f \in C^{1}(\mathbb{R})$ and $u \notin H^{1}\left(B_{1}\right)$ be a semi-stable radial solution of (1.1). Then there exist $K>0$ and $0<r_{0}<1$ such that

$$
\int_{r / 2}^{r} \frac{d s}{u_{r}(s)^{2}} \leq K r^{N+2 \sqrt{N-1}-1} \quad \forall r \in\left(0, r_{0}\right)
$$

Proof. Consider $a$ and $\eta_{0}$ of Lemma 2.3. From Lemma 2.2 we can choose $0<r_{0}<a$ such that $u_{r}$ does not vanish in $\left(0, r_{0}\right]$. We now fix $r \in\left(0, r_{0}\right)$ and consider the function

$$
\eta(t)= \begin{cases}\frac{r^{\sqrt{N-1}}}{\int_{r / 2}^{r} \frac{d s}{u_{r}(s)^{2}}} \int_{r / 2}^{t} \frac{d s}{u_{r}(s)^{2}} & \text { if } r / 2 \leq t \leq r, \\ t^{\sqrt{N-1}} & \text { if } r<t \leq a, \\ a^{\sqrt{N-1}} \eta_{0}(t) & \text { if } a<t \leq 1 / 2 .\end{cases}
$$

Applying Lemma 2.1 (with $r_{1}=r / 2$ and $r_{2}=1 / 2$ ) we obtain

$$
\begin{gathered}
0 \leq \int_{r / 2}^{1 / 2} t^{N-1} u_{r}(t)^{2}\left(\eta^{\prime}(t)^{2}-\frac{N-1}{t^{2}} \eta(t)^{2}\right) d t \\
=\int_{r / 2}^{r} t^{N-1} u_{r}(t)^{2}\left(\eta^{\prime}(t)^{2}-\frac{N-1}{t^{2}} \eta(t)^{2}\right) d t \\
+a^{2 \sqrt{N-1}} \int_{a}^{1 / 2} t^{N-1} u_{r}(t)^{2}\left(\eta_{0}^{\prime}(t)^{2}-\frac{N-1}{t^{2}} \eta_{0}(t)^{2}\right) d t \\
\leq r^{N-1} \int_{r / 2}^{r} u_{r}(t)^{2} \eta^{\prime}(t)^{2} d t+a^{2 \sqrt{N-1}} \int_{a}^{1 / 2} t^{N-1} u_{r}(t)^{2}\left(\eta_{0}^{\prime}(t)^{2}-\frac{N-1}{t^{2}} \eta_{0}(t)^{2}\right) d t \\
=r^{N-1} \frac{r^{2 \sqrt{N-1}}}{\int_{r / 2}^{r} \frac{d s}{u_{r}(s)^{2}}}+a^{2 \sqrt{N-1}} \int_{a}^{1 / 2} t^{N-1} u_{r}(t)^{2}\left(\eta_{0}^{\prime}(t)^{2}-\frac{N-1}{t^{2}} \eta_{0}(t)^{2}\right) d t .
\end{gathered}
$$

This gives

$$
-a^{2 \sqrt{N-1}} \int_{a}^{1 / 2} t^{N-1} u_{r}(t)^{2}\left(\eta_{0}^{\prime}(t)^{2}-\frac{N-1}{t^{2}} \eta_{0}(t)^{2}\right) d t \leq \frac{r^{N+2 \sqrt{N-1}-1}}{\int_{r / 2}^{r} \frac{d s}{u_{r}(s)^{2}}}
$$

which is the desired conclusion for 


$$
K=\left(-a^{2 \sqrt{N-1}} \int_{a}^{1 / 2} t^{N-1} u_{r}(t)^{2}\left(\eta_{0}^{\prime}(t)^{2}-\frac{N-1}{t^{2}} \eta_{0}(t)^{2}\right) d t\right)^{-1},
$$

which is a positive number, from Lemma 2.3.

Lemma 2.5. Let $N \geq 2, f \in C^{1}(\mathbb{R})$ and $u \notin H^{1}\left(B_{1}\right)$ be a semi-stable radial solution of (1.1). Then there exist $M^{\prime}>0$ and $0<r_{0}<1$ such that

$$
|u(r)-u(r / 2)| \geq M^{\prime} r^{-N / 2-\sqrt{N-1}+2} \quad \forall r \in\left(0, r_{0}\right) .
$$

Proof. Take the same constant $0<r_{0}<1$ of Lemma 2.4. Fix $r \in\left(0, r_{0}\right)$ and consider the functions:

$$
\begin{aligned}
& \alpha(s)=\left|u_{r}(s)\right|^{-\frac{2}{3}}, \quad s \in(r / 2, r) . \\
& \beta(s)=\left|u_{r}(s)\right|^{\frac{2}{3}}, \quad s \in(r / 2, r) .
\end{aligned}
$$

By Lemma 2.4 we have

$$
\|\alpha\|_{L^{3}(r / 2, r)} \leq K^{\frac{1}{3}} r^{\frac{N+2 \sqrt{N-1}-1}{3}}
$$

for a constant $K>0$ not depending on $r \in\left(0, r_{0}\right)$. On the other hand, since $u_{r}$ does not vanish in $(0, a]$, it follows

$$
\|\beta\|_{L^{3 / 2}(r / 2, r)}=|u(r)-u(r / 2)|^{\frac{2}{3}} .
$$

Applying Hölder inequality to functions $\alpha$ and $\beta$ we deduce

$r / 2=\int_{r / 2}^{r} \alpha(s) \beta(s) d s \leq\|\alpha\|_{L^{3}(r / 2, r)}\|\beta\|_{L^{3 / 2}(r / 2, r)} \leq K^{\frac{1}{3}} r^{\frac{N+2 \sqrt{N-1}-1}{3}}|u(r)-u(r / 2)|^{\frac{2}{3}}$,

which is the desired conclusion for $M^{\prime}=2^{-3 / 2} K^{-1 / 2}$.

Proof of Theorem 1.1. Consider the numbers $M^{\prime}>0$ and $0<r_{0}<1$ of Lemma 2.5. It is easily seen that for every $r \in\left(0, r_{0}\right)$ there exist an integer $m \geq 0$ and $r_{0} / 2 \leq z<r_{0}$ such that $r=z / 2^{m}$. From the monotonicity of $u$ in $\left(0, r_{0}\right)$ it follows that

$$
|u(r)| \geq|u(z)-u(r)|-|u(z)|=\sum_{k=0}^{m-1}\left|u\left(\frac{z}{2^{k}}\right)-u\left(\frac{z}{2^{k+1}}\right)\right|-|u(z)|
$$

- Case $N=2$. We have that $-N / 2-\sqrt{N-1}+2=0$. Hence, applying Lemma 2.5 and (2.1) we obtain

$$
|u(r)| \geq M^{\prime} m-|u(z)|=\frac{M^{\prime}(\log z-\log r)}{\log 2}-|u(z)|
$$


where $M^{\prime}>0$ does not depend on $r \in\left(0, r_{0}\right)$. Since $z \in\left[r_{0} / 2, r_{0}\right)$ and $u$ is continuous the above inequality is of the type

$$
|u(r)| \geq M_{1}|\log r|-M_{2} \quad \forall r \in\left(0, r_{0}\right),
$$

for certain $M_{1}, M_{2}>0$. Taking a smaller $0<r_{0}<1$ if necessary, the theorem is proved in this case.

- Case $N \geq 3$. We have that $-N / 2-\sqrt{N-1}+2<0$. Thus, applying again Lemma 2.5 and (2.1) we deduce

$$
\begin{gathered}
|u(r)| \geq \sum_{k=0}^{m-1} M^{\prime}\left(\frac{z}{2^{k}}\right)^{-N / 2-\sqrt{N-1}+2}-|u(z)| \\
=M^{\prime}\left(\frac{r^{-N / 2-\sqrt{N-1}+2}-z^{-N / 2-\sqrt{N-1}+2}}{2^{N / 2+\sqrt{N-1}-2}-1}\right)-|u(z)|,
\end{gathered}
$$

which is an inequality of the type $|u(r)| \geq M_{1} r^{-N / 2-\sqrt{N-1}+2}-M_{2}, \forall r \in$ $\left(0, r_{0}\right)$, for certain $M_{1}, M_{2}>0$. The proof is complete as the previous case.

\section{Proof of Theorem 1.2,}

If $N=2$, then $\left(-r u_{r}(r)\right)^{\prime}=r f(u(r)) \geq 0$ for every $r \in(0,1]$. Since $-r u_{r}(r)$ is nonnegative for small $r$, it is deduced that $\lim _{r \rightarrow 0}\left(-r u_{r}(r)\right)=$ $\alpha$, for some $\alpha \in[0, \infty)$. This implies $\lim _{r \rightarrow 0} u(r) /|\log r|=\alpha$. Applying Theorem 1.1 we deduced $\alpha>0$, which is our claim for $N=2$.

If $N \geq 3$, then $\left(-r^{N-1} u_{r}(r)\right)^{\prime}=r^{N-1} f(u(r)) \geq 0$ for every $r \in(0,1]$. Since $-r^{N-1} u_{r}(r)$ is nonnegative for small $r$, it is deduced that $-r^{N-1} u_{r}(r)$ is a nonnegative nondecreasing function and then $r^{N-1}\left|u_{r}(r)\right|=-r^{N-1} u_{r}(r) \leq$ $-u_{r}(1)$, following the second inequality of (ii) for $M_{2}=-u_{r}(1)$. (Note that we have used neither the semi-stability of $u$ nor $\left.u \notin H^{1}\left(B_{1}\right)\right)$. To prove the first inequality of (ii), let us observe that since $-r^{N-1} u_{r}$ is a nonnegative nondecreasing function then $r^{2 N-2} u_{r}^{2}$ is nondecreasing. Then applying Lemma 2.4 we have that there exist $K>0$ and $0<r_{0}<1$ such that

$$
\begin{gathered}
K r^{N+2 \sqrt{N-1}-1} \geq \int_{r / 2}^{r} \frac{d s}{u_{r}(s)^{2}}=\int_{r / 2}^{r} \frac{s^{2 N-2}}{s^{2 N-2} u_{r}(s)^{2}} d s \\
\geq \frac{1}{r^{2 N-2} u_{r}(r)^{2}} \int_{r / 2}^{r} s^{2 N-2} d s=\frac{\left(1-2^{1-2 N}\right) r}{(2 N-1) u_{r}(r)^{2}}
\end{gathered}
$$

for every $r \in\left(0, r_{0}\right)$, which is the desired conclusion in the interval $\left(0, r_{0}\right)$ for $M_{1}=\left(\left(1-2^{1-2 N}\right) /((2 N-1) K)\right)^{1 / 2}$. To finish the proof it remains to show that $u_{r}(r)<0$ for every $0<r \leq 1$. Indeed, if $u_{r}\left(r^{\prime}\right) \geq 0$ for some $0<r^{\prime} \leq 1$ then, from the nonnegativeness and the monotonicity of $-r^{N-1} u_{r}(r)$ in $(0,1]$, it is deduced that $-r^{N-1} u_{r}(r)=0$ for every $0<r \leq r^{\prime}$. Hence $u$ is constant in $\left(0, r^{\prime}\right]$, a contradiction. 


\section{SEmi-Stable RAdial WEAK SOlUtions in A BALL}

The following lemma gives a characterization of radial weak solutions of (1.2) and will be useful to prove Theorem 1.3 ,

Lemma 3.1. Let $\Omega=B_{1}, f \in C(\mathbb{R})$ and $u$ be a radial function in $\overline{B_{1}}$. Then $u$ is a weak solution of (1.2) if and only if the following holds:

(i) $u \in C^{2}(0,1], u(1)=0$ and $-\Delta u(x)=f(u(x))$ pointwise in $\overline{B_{1}} \backslash\{0\}$.

(ii) $f(u) \in L^{1}\left(B_{1}\right)$.

(iii) $\lim _{r \rightarrow 0} r^{N-1} u_{r}(r)=0$.

Proof. Let us prove first the necessary conditions. Suppose that $u$ is a radial weak solution of (1.2). Then it is well known that

$$
u(r)=-\int_{r}^{1}\left(\frac{u_{r}(1)+\int_{t}^{1} s^{N-1} f(u(s)) d s}{t^{N-1}}\right) d t
$$

and (i) is proved. On the other hand since $f(u) \delta \in L^{1}\left(B_{1}\right)$ then $f(u) \in$ $L^{1}\left(B_{1 / 2}\right)$. Taking into account that $f(u)$ is continuous in $\overline{B_{1}} \backslash B_{1 / 2}$, (ii) is proved. To prove (iii), consider $\zeta \in C^{2}\left(\overline{B_{1}}\right)$ satisfying $\zeta=0$ on $\partial B_{1}$ and $\zeta=1$ in $B_{1 / 2}$. Applying (1.3) we deduce

$$
\begin{gathered}
0=\int_{B_{1}}(u \Delta \zeta+f(u) \zeta) d x=\lim _{r \rightarrow 0} \int_{B_{1} \backslash \overline{B_{r}}}(u \Delta \zeta+f(u) \zeta) d x \\
=\lim _{r \rightarrow 0} \int_{B_{1} \backslash \overline{B_{r}}}(u \Delta \zeta-\zeta \Delta u) d x=\lim _{r \rightarrow 0} \int_{\partial\left(B_{1} \backslash \overline{B_{r}}\right)}(u \nabla \zeta-\zeta \nabla u) \\
=\lim _{r \rightarrow 0}\left(-\omega_{N} r^{N-1} u_{r}(r)\right),
\end{gathered}
$$

and (iii) follows.

Suppose now that (i), (ii) and (iii) hold for a radial function $u$ defined in $\overline{B_{1}}$. From (iii) it is deduced that $\lim _{r \rightarrow 0} u(r) /|\log r|=0$ for $N=2$, while $\lim _{r \rightarrow 0} u(r) r^{N-2}=0$ for $N \geq 3$. In all the cases we have $\lim _{r \rightarrow 0} r^{N-1} u(r)=$ 0 , which gives $r^{N-1} u(r) \in L^{\infty}(0,1)$ and then $u \in L^{1}\left(B_{1}\right)$. On the other hand (ii) clearly implies $f(u) \delta \in L^{1}\left(B_{1}\right)$. What is left to show is (1.3). To this end, consider $\zeta \in C^{2}\left(\overline{B_{1}}\right)$ satisfying $\zeta=0$ on $\partial B_{1}$. Applying (i) and (ii) we obtain that

$$
\begin{gathered}
\int_{B_{1}}(u \Delta \zeta+f(u) \zeta) d x=\lim _{r \rightarrow 0} \int_{B_{1} \backslash \overline{B_{r}}}(u \Delta \zeta+f(u) \zeta) d x \\
=\lim _{r \rightarrow 0} \int_{B_{1} \backslash \overline{B_{r}}}(u \Delta \zeta-\zeta \Delta u) d x=\lim _{r \rightarrow 0} \int_{\partial\left(B_{1} \backslash \overline{B_{r}}\right)}(u \nabla \zeta-\zeta \nabla u)= \\
\lim _{r \rightarrow 0} \int_{\partial B_{r}}(u \nabla \zeta-\zeta \nabla u) .
\end{gathered}
$$

Consider $M>0$ such that $|\zeta|,|\nabla \zeta| \leq M$ in $\overline{B_{1}}$. Applying $\lim _{r \rightarrow 0} r^{N-1} u(r)=$ 0 and $\lim _{r \rightarrow 0} r^{N-1} u_{r}(r)=0$ the proof is complete by observing that 


$$
\begin{gathered}
\left|\int_{\partial B_{r}}(u \nabla \zeta-\zeta \nabla u)\right| \leq \int_{\partial B_{r}}(|u \nabla \zeta|+|\zeta \nabla u|) \leq M \int_{\partial B_{r}}(|u|+|\nabla u|) \\
=M \omega_{N} r^{N-1}\left(|u(r)|+\left|u_{r}(r)\right|\right) \rightarrow 0 \text { as } r \rightarrow 0 .
\end{gathered}
$$

Remark 1. We can apply this characterization to the radial functions $u(r)=$ $r^{-2 /(p-1)}-1,(p>1)$ mentioned in the Introduction. We have that $u$ is a solution of (1.1) for $f(u)=2(N p-2 p-N) /(p-1)^{2}(1+u)^{p}$. Applying Lemma 3.1, we check at once that $u$ is a radial weak solution of (1.2) if and only if $N \geq 3$ and $p>N /(N-2)$.

Proof of Theorem 1.3. Suppose that $u \notin H^{1}\left(B_{1}\right)$. Applying Theorem 1.1 we have that there exist $M>0$ and $0<r_{0}<1$ such that $|u(r)| \geq M|\log r|$ for every $r \in\left(0, r_{0}\right)$. On the other hand, since $u$ is a radial weak solution of (1.2) we could apply (iii) of Lemma (3.1) and obtain $\lim _{r \rightarrow 0} r u_{r}(r)=0$. In particular $\lim _{r \rightarrow 0} u(r) /|\log r|=0$, a contradiction.

Thus $u$ is an energy solution (i.e. $u \in H^{1}\left(B_{1}\right)$ ). It is known (see [4]) that $u \in L^{\infty}\left(B_{1}\right)$ and then, by standard regularity arguments, $u \in C^{2}\left(\overline{B_{1}}\right)$.

\section{REFERENCES}

[1] H. Brezis, T. Cazenave, Y. Martel, A. Ramiandrisoa, Blow up for $u_{t}-\Delta u=g(u)$ revisited, Adv. Differential Equations 1, 73-90 (1996).

[2] H. Brezis, J. L. Vázquez, Blow-up solutions of some nonlinear elliptic problems, Rev. Mat. Univ. Complut. Madrid 10, 443-469 (1997).

[3] X. Cabré, Regularity of minimizers of semilinear elliptic problems up to dimension 4, Comm. Pure Appl. Math. 63, 1362-1380 (2010).

[4] X. Cabré, A. Capella, Regularity of radial minimizers and extremal solutions of semilinear elliptic equations, J. Funct. Anal. 238, 709-733 (2006).

[5] X. Cabré, X. Ros-Oton, Regularity of stable solutions up to dimension 7 in domains of double revolution, Comm. Partial Differential Equations 38, 135-154 (2013).

[6] X. Cabré, M. Sanchón, Geometric-type Sobolev inequalities and applications to the regularity of minimizers, J. Funct. Anal. 264, 303-325 (2013).

[7] M. G. Crandall, P. H. Rabinowitz, Some continuation and variational methods for positive solutions of nonlinear elliptic eigenvalue problems, Arch. Rational Mech. Anal. 58, 207-218 (1975).

[8] L. Dupaigne, Stable solutions of elliptic partial differential equations, Chapman \& Hall/CRC Monographs and Surveys in Pure and Applied Mathematics 143, Chapman \& Hall/CRC, Boca Raton, FL (2011).

[9] D. D. Joseph, T. S. Lundgren, Quasilinear Dirichlet problems driven by positive sources, Arch. Rational Mech. Anal. 49, 241-269 (1973).

[10] E. Giusti, Minimal surfaces and functions of bounded variation, Monographs in Mathematics 80, Birkhäuser Verlag, Basel (1984).

[11] F. Mignot, J.-P. Puel, Sur une classe de problèmes non linéaires avec non linéairité positive, croissante, convexe, Comm. Partial Differential Equations 5, 791-836 (1980).

[12] G. Nedev, Regularity of the extremal solution of semilinear elliptic equations, C. R. Acad. Sci. Paris Sér. I Math. 330, 997-1002 (2000).

[13] G. Nedev, Extremal solutions of semilinear elliptic equations, Preprint (2001). 
[14] S. Villegas, Boundedness of extremal solutions in dimension 4, Adv. Math. 235, 126133 (2013).

[15] S. Villegas, Dichotomy of stable radial solutions of $-\Delta u=f(u)$ outside a ball, arXiv:1404.1722v1 (2014).

Departamento de Análisis Matemático, Universidad de Granada, 18071 Granada, SPAIN.

E-mail address: svillega@ugr.es 